\title{
Resonance-Size Parameter Relationship and Dynamics of an AFM Subjected to Multimode Excitation and Based on the Modified Couple Stress Theory
}

\author{
Shueei-Muh Lin (i) and Ching-Yao Chang \\ Mechanical Engineering Department, Kun Shan University, Tainan 710-03, Taiwan \\ Correspondence should be addressed to Shueei-Muh Lin; smlin45@gmail.com
}

Received 24 March 2019; Accepted 20 May 2019; Published 16 June 2019

Academic Editor: Weizhong Dai

Copyright (C) 2019 Shueei-Muh Lin and Ching-Yao Chang. This is an open access article distributed under the Creative Commons Attribution License, which permits unrestricted use, distribution, and reproduction in any medium, provided the original work is properly cited.

\begin{abstract}
The mathematical model of AFM probe subjected to multimode excitation based on the modified couple stress theory is presented. The semianalytical solution of the system is proposed. The transient behavior and response spectrum of AFM probe subjected to multimode excitation are investigated. It is very helpful to predict the nanotopography and surface properties based on the response of multimodes excitation. The effects of the root excitation, size parameter, and interacting distance on the response spectrum and frequency shift are investigated. The resonant frequency relation of the two systems with different size parameters is discovered and expressed in a formula. The natural frequencies predicted via the formula and those determined by the semianalytical method are significantly consistent.
\end{abstract}

\section{Introduction}

Atomic force microscopy (AFM) is a powerful device for scanning the atomic-scale topography and property of a sample's surface [1].

In convention, the literatures investigated the steady behavior of AFM probe [2-9]. Definitely, the investigation of the amplitude and phase behavior in the transient regime will increase significantly the speed of imaging and accuracy. Santos and Gaderlab [10] investigated the tip-sample force in transient behavior. Sahoo et al. [11] increased the speed of imaging and control of AFM by studying the transient motion of cantilever signal. Chang et al. [12] presented a new algorithm for high speed AFM imaging of biopolymers by investigating the transition from transient part to steady state regime. Payam [9] studied the transient behavior of tapping mode-AFM in the mass-spring-damper model.

Some literatures [13-16] investigated the multifrequency excitation of FM-AFM systems in fast force spectroscopy and material characterization at the atomic scale as well. The topography and material properties of sample's surface can be measured by using AFM subjected to the simultaneous multimodes excitations. The amplitude signal of the first mode is used to image the surface topography. The phase shift signal of the second mode is usually used to map changes in material properties of the sample's surface [17-19].

In convention, the structure's size effect is not considered. Several literatures [20-23] found that the size-dependent effect on the dynamic behavior of the structures in the order of microns or submicrons is significant. Some nonclassical theories with size dependency are the couple stress theory [16, $24,25]$, the strain gradient theory [26], the nonlocal theory [27], and the surface elasticity model [28]. Mindlin and Tiersten [24] and Toupin [25] presented the classical couple stress theory with the gradients of rotation and displacement. Yang et al. [29] modified the classical stress theory (MCST) by considering that the density of strain energy is a function of the strain and curvature tensors.

Several literatures [30-34] investigated the size effect on the behavior of beam. Ansari et al. [35] investigated the size-dependent resonant frequency and flexural sensitivity of AFM based on the modified strain gradient theory by using the force gradient method. However, it is well known that the 


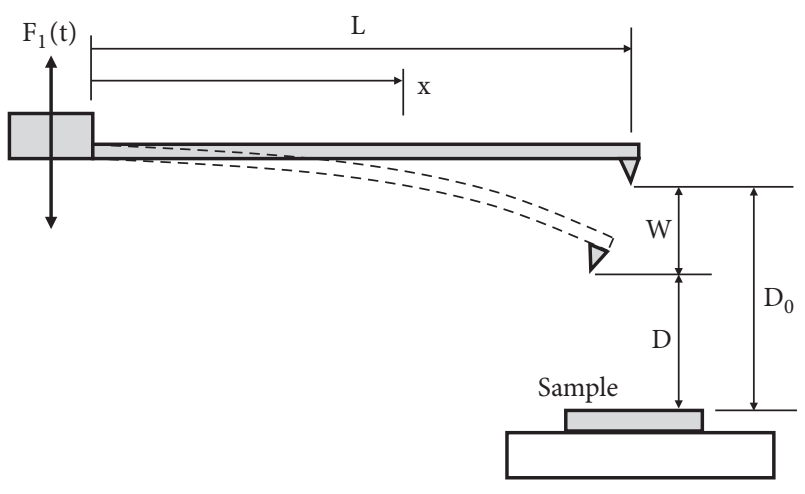

FIGURE 1: Geometry and coordinate system of AFM.

interpretation of frequency shift by using the force gradient method is unsatisfactory [4].

So far, no literature is devoted to investigate the transient behavior of an AFM probe subjected to multimodes excitations based on the modified couple stress theory. In this study, the mathematical model of AFM probe subjected to two-mode excitation and the van der Waals force based on the modified couple stress theory is constructed. The semianalytical method is presented. The effects of several parameters on the transient behavior are investigated.

\section{Dynamic System Subjected to Multi-Mode Excitation}

2.1. Governing Equation and Boundary Conditions. Consider the transient response of atomic force microscopy excited at the root measuring the interatomic van der Waals force, as shown in Figure 1. The multimode harmonic excitation is presented, as shown in Figure 1. The governing equation is

$$
\left(E I+\mu A \ell_{c}^{2}\right) \frac{\partial^{4} W}{\partial x^{4}}+\rho A \frac{\partial^{2} W}{\partial t^{2}}=0, \quad 0<x<L
$$

The boundary conditions are as follows.

$$
\begin{aligned}
& \text { At } \mathrm{x}=0: \\
& \qquad \begin{aligned}
W & =W_{e 1} \sin \Omega_{e 1} t+W_{e 2} \sin \Omega_{e 2} t \equiv F_{1}(t), \\
\frac{\partial W}{\partial x} & =0
\end{aligned}
\end{aligned}
$$

At $\mathrm{x}=\mathrm{L}$ :

$$
\begin{aligned}
\frac{\partial^{2} W}{\partial x^{2}} & =0 \\
\left(E I+\mu A \ell_{c}^{2}\right)\left(\frac{\partial^{3} W}{\partial x^{3}}\right)-m_{t} \frac{\partial^{2} W}{\partial t^{2}} & =F_{v}
\end{aligned}
$$

where $A$ and $I$ denote the cross-sectional area and the area moment of inertia, respectively. $\rho$ is the mass density per unit volume, $m_{t}$ the tip mass, $W$ the flexural displacement, $E$ Young's modulus, $x$ the coordinate along the beam, $L$ the length of the beam, and $\ell_{c}$ the material length scale parameter. The van der Waals force [11] is

$$
F_{v}=-\frac{A_{H} R}{6 D^{2}}
$$

in which $A_{H}$ is the Hamaker constant, $D$ the tip-surface distance, and $R$ the tip radius.

In terms of the following dimensionless quantities:

$$
\begin{aligned}
& b(\xi)=\frac{E(x) I(x)}{E(0) I(0)}, \\
& c_{v}=\frac{A_{H} R L^{3}}{E(0) I(0) L_{0}^{3}}, \\
& \bar{D}=\frac{D}{L_{0}}, \\
& f_{v}=\frac{-c_{v}}{6\left(\bar{D}_{0}-w\right)^{2}}=\frac{-c_{v}}{6 \bar{D}^{2}}, \\
& m=\frac{\rho(x) A(x)}{\rho(0) A(0)}, \\
& w(\xi, \tau)=\frac{W(x, t)}{L_{0}}, \\
& w_{e 1}=\frac{W_{e 1}}{L_{0}}, \\
& w_{e 2}=\frac{W_{e 2}}{L_{0}}, \\
& \bar{m}_{t}=\frac{m_{t}}{\rho(0) A(0) L} \text {, } \\
& \xi=\frac{x}{L}, \\
& \tau=\frac{t}{L^{2}} \sqrt{\frac{E(0) I(0)}{\rho(0) A(0)}}, \\
& \omega_{e i}=\Omega_{e i} L^{2} \sqrt{\frac{\rho A}{E I}} \\
& \delta=\frac{\mu A \ell_{c}^{2}}{E I}
\end{aligned}
$$

where $L_{0}$ is the characteristic length. A small value of $L_{0}$ is introduced to avoid the numerical transaction error. The dimensionless governing differential equation of the system is

$$
(1+\delta) \frac{\partial^{4} w}{\partial \xi^{4}}+\frac{\partial^{2} w}{\partial \tau^{2}}=0
$$

The associated boundary conditions are as follows.

At $\xi=0$ :

$$
\begin{aligned}
w & =w_{e 1} \sin \omega_{e 1} \tau+w_{e 2} \sin \omega_{e 2} \tau \equiv f_{1}(\tau) \\
\frac{\partial w}{\partial \xi} & =0
\end{aligned}
$$




$$
\text { At } \xi=1 \text { : }
$$

$$
\begin{gathered}
\frac{\partial^{2} w}{\partial \xi^{2}}=0 \\
(1+\delta) \frac{\partial^{3} w}{\partial \xi^{3}}-\bar{m}_{t} \frac{\partial^{2} w}{\partial \tau^{2}}=f_{v}(w(1, \tau))
\end{gathered}
$$

The initial conditions are

$$
\begin{aligned}
w(\xi, 0) & =0, \\
\frac{\partial w(\xi, 0)}{\partial \tau} & =0
\end{aligned}
$$

2.2. Solution Method. Because the system composed of (8)(13) is nonlinear and the boundary condition is timedependent, it is difficult to directly solve. The semianalytical method is presented here.

2.2.1. Linearization of System. Due to its complexity, the nonlinear general system is approximated by infinite linearized subsystems. At first, the time variable $\tau$ is divided into infinite sections and the dynamic performance of the system is derived step by step. The methodology is described here. The tip displacement is presented by the Taylor series as

$$
\begin{aligned}
\widetilde{w}(1, \tau)= & w\left(1, \tau_{i}\right)+\frac{\partial w\left(1, \tau_{i}\right)}{\partial \tau}\left(\tau-\tau_{i}\right) \\
& +\frac{1}{2} \frac{\partial^{2} w\left(1, \tau_{i}\right)}{\partial \tau^{2}}\left(\tau-\tau_{i}\right)^{2}+\Delta\left(\tau_{i+1}\right), \\
\frac{\partial w(1, \tau)}{\partial \tau} \approx & \frac{\partial w\left(1, \tau_{i}\right)}{\partial \tau}+\frac{\partial^{2} w\left(1, \tau_{i}\right)}{\partial \tau^{2}}\left(\tau-\tau_{i}\right) \\
\frac{\partial^{2} w(1, \tau)}{\partial \tau^{2}} \approx & {\left[\frac{\partial^{2} w\left(1, \tau_{i}\right)}{\partial \tau^{2}}\right], \text { for } \tau_{i}<\tau<\tau_{i+1} }
\end{aligned}
$$

where $\Delta\left(\tau_{i+1}\right)$ is an unknown transaction difference to be determined. Based on this relation and the following methods one can determine the displacement $\mathrm{w}(\xi, \tau)$. Moreover, the error of the tip displacement must approach zero:

$$
\operatorname{Error}\left(\tau_{i+1}\right)=\left|w\left(1, \tau_{i+1}\right)-\widetilde{w}\left(1, \tau_{i+1}\right)\right|<\varepsilon \longrightarrow 0
$$

Substituting (14) into the nonlinear nonhomogeneous boundary condition (12), the linearized time-dependent boundary condition is obtained:

$$
\begin{aligned}
\frac{\partial^{3} w}{\partial \xi^{3}} & \approx \frac{1}{(1+\delta)}\left[\bar{m}_{t}\left[\frac{\partial^{2} w\left(1, \tau_{i}\right)}{\partial \tau^{2}}\right]+f_{v}(\widetilde{w}(1, \tau))\right] \\
& \triangleq f_{2}(\tau, \Delta), \quad \text { for } \tau_{i}<\tau<\tau_{i+1}
\end{aligned}
$$

So far, the nonlinear system is linearized in each time domain. The approximated system can be exactly solved by the solution method presented in the following sections. Therefore, the overall displacement can be derived step by step.
So far, the linearized subsystem in the time domain $\left(\tau_{i}, \tau_{i+1}\right)$ can be expressed as follows:

$$
(1+\delta) \frac{\partial^{4} w}{\partial \xi^{4}}+\frac{\partial^{2} w}{\partial \tau^{2}}=0, \quad \text { for } \tau_{i}<\tau<\tau_{i+1}
$$

The associated time-dependent boundary conditions are as follows.

$$
\begin{aligned}
& \text { At } \xi=0 \text { : } \\
& w=w_{e 1} \sin \omega_{e 1} \tau+w_{e 2} \sin \omega_{e 2} \tau \equiv f_{1}(\tau) \\
& \frac{\partial w}{\partial \xi}=0
\end{aligned}
$$

At $\xi=1$ :

$$
\begin{aligned}
& \frac{\partial^{2} w}{\partial \xi^{2}}=0 \\
& \frac{\partial^{3} w}{\partial \xi^{3}}=f_{2}(\tau, \Delta), \quad \text { for } \tau_{i}<\tau<\tau_{i+1}
\end{aligned}
$$

\subsubsection{Solution Method of Linearized Subsystem}

(A) Transform of Variable. The linearized subsystem includes two nonhomogeneous time-dependent boundary conditions. Solving this subsystem, the following transformation of variable is considered:

$$
w(\xi, \tau)=v(\xi, \tau)+\sum_{i=1}^{2} g_{i}(\xi) f_{i}(\tau), \quad \text { for } \tau_{i}<\tau<\tau_{i+1}
$$

Substituting relation (22) into (17)-(21), two following subsystems are obtained. The first subsystem is expressed in terms of the transformed variable $v(\xi, \tau)$ as follows: the transformed governing equation is

$$
(1+\delta) \frac{\partial^{4} v}{\partial \xi^{4}}+\frac{\partial^{2} v}{\partial \tau^{2}}=-\sum_{i=1}^{2} g_{i}(\xi) \frac{d^{2} f_{i}}{d \tau^{2}}
$$

$$
\text { for } \tau_{i}<\tau<\tau_{i+1}
$$

The associated boundary conditions are as follows.

At $\xi=0$ :

$$
\begin{array}{r}
v(0, \tau)=0, \\
\frac{\partial v(0, \tau)}{\partial \xi}=0
\end{array}
$$

At $\xi=1$ :

$$
\begin{aligned}
& \frac{\partial^{2} v(1, \tau)}{\partial \xi^{2}}=0, \\
& \frac{\partial^{3} v(1, \tau)}{\partial \xi^{3}}=0
\end{aligned}
$$

The corresponding initial conditions are

$$
v(\xi, 0)=-\sum_{i=1}^{2} g_{i}(\xi) f_{i}(0)
$$




$$
\frac{\partial v(\xi, 0)}{\partial \tau}=-\sum_{i=1}^{2} g_{i}(\xi) \frac{d f_{i}(0)}{d \tau}
$$

The second subsystem is expressed in terms of the shifting function $g_{1}(\xi)$. The second transformed governing equation is

$$
\frac{d^{4} g_{1}}{d \xi^{4}}=0, \quad 0<\xi<1
$$

At $\xi=0$ :

$$
\begin{aligned}
g_{1}(0) & =1, \\
\frac{d g_{1}(0)}{d \xi} & =0
\end{aligned}
$$

At $\xi=1$ :

$$
\begin{aligned}
& \frac{d^{2} g_{1}(1)}{d \xi^{2}}=0 \\
& \frac{d^{3} g_{1}(1)}{d \xi^{3}}=0
\end{aligned}
$$

The third subsystem is expressed in terms of the shifting function $g_{2}(\xi)$. The third transformed governing equation is

$$
\frac{d^{4} g_{2}}{d \xi^{4}}=0, \quad 0<\xi<1
$$

At $\xi=0$ :

$$
\begin{gathered}
g_{2}(0)=0, \\
\frac{d g_{2}(0)}{d \xi}=0
\end{gathered}
$$

At $\xi=1$ :

$$
\begin{aligned}
& \frac{d^{2} g_{2}(1)}{d \xi^{2}}=0 \\
& \frac{d^{3} g_{2}(1)}{d \xi^{3}}=1
\end{aligned}
$$

The solutions of the two shifting functions $\left\{g_{1}, g_{2}\right\}$ are easily discovered as follows:

$$
\begin{aligned}
& g_{1}(\xi)=1 \\
& g_{2}(\xi)=-\frac{1}{2} \xi^{2}+\frac{1}{6} \xi^{3}
\end{aligned}
$$

\section{(B) Solution of the First Subsystem}

(B-1) Orthogonality of Eigenfunctions. The frequency equation of the first subsystem composed of (23)-(27) is

$$
\cosh \sqrt{\bar{\omega}} \cos \sqrt{\bar{\omega}}+1=0
$$

where $\bar{\omega}=\omega / \sqrt{(1+\delta)}$. The $\mathrm{n} t h$ mode shapes are

$$
\begin{aligned}
y_{n}= & {\left[\sinh \sqrt{\bar{\omega}_{n}}+\sin \sqrt{\bar{\omega}_{n}}\right] } \\
\cdot & {\left[\cosh \sqrt{\bar{\omega}_{n}} \xi-\cos \sqrt{\bar{\omega}_{n}} \xi\right] } \\
& -\left[\cosh \sqrt{\bar{\omega}_{n}}+\cos \sqrt{\bar{\omega}_{n}}\right] \\
& \cdot\left[\sinh \sqrt{\bar{\omega}_{n}} \xi-\sin \sqrt{\bar{\omega}_{n}} \xi\right]
\end{aligned}
$$

which is the same as that given by Rao [36]. The orthogonality of the eigenfunctions is presented as follows:

$$
\int_{0}^{1} y_{n} y_{k} d \xi= \begin{cases}0, & n \neq k \\ \delta_{n}, & n=k\end{cases}
$$

(B-2) Modes Superposition Method. Based on the orthogonality conditions (44), the mode superposition method is used to derive the solution of the first subsystem composed of (23)(27). The transformed variable is expressed in terms of the eigenfunctions

$$
v(\xi, \tau)=\sum_{n=1}^{\infty} y_{n}(\xi) T_{n}(\tau)
$$

Substituting (45) into (23) and multiplying it by $y_{n}$ and integrating it from 0 to 1 , one obtains

$$
\begin{array}{r}
\frac{d^{2} T_{m}(\tau)}{d \tau^{2}}+\omega_{m}^{2} T_{m}(\tau)=\frac{1}{\delta_{m}} \int_{0}^{1} f_{t}(\xi, \tau) y_{m} d \xi \\
\equiv \widetilde{F}_{m}(\tau), \text { for } \tau_{i}<\tau<\tau_{i+1} ; m=1,2, \ldots
\end{array}
$$

where

$$
\begin{gathered}
f_{t}(\xi, t)=\left[\omega_{1}^{2}\left(w_{1} \sin \omega_{1} \tau\right)+\omega_{2}^{2}\left(w_{2} \sin \omega_{2} \tau\right)\right] \\
-\left(-\frac{1}{2} \xi^{2}+\frac{1}{6} \xi^{3}\right) \frac{1}{(1+\delta)} \frac{d^{2} \widetilde{f}_{t s}(w(1, \tau))}{d \tau^{2}} \\
\widetilde{F}_{n}(\tau)=\frac{1}{\delta_{n}}\left\{\alpha_{n}\left[\omega_{1}^{2}\left(w_{1} \sin \omega_{1} \tau\right)+\omega_{2}^{2}\left(w_{2} \sin \omega_{2} \tau\right)\right]\right. \\
\left.-\frac{\beta_{n}}{(1+\delta)} \frac{d^{2} f_{v}}{d \tau^{2}}\right\}
\end{gathered}
$$

in which

$$
\begin{aligned}
\alpha_{n} & =\frac{-2}{\sqrt{\bar{\omega}_{n}}}\left[1+\cosh \sqrt{\bar{\omega}_{n}} \cos \sqrt{\bar{\omega}_{n}}-\cosh \sqrt{\bar{\omega}_{n}}\right. \\
& \left.-\cos \sqrt{\bar{\omega}_{n}}\right] \\
\beta_{n} & =\left(\gamma_{n 1}+\gamma_{n 2}\right)\left(\sinh \sqrt{\bar{\omega}_{n}}+\sin \sqrt{\bar{\omega}_{n}}\right)-\left(\gamma_{n 3}\right. \\
& \left.+\gamma_{n 4}\right)\left(\cosh \sqrt{\bar{\omega}_{n}}+\cos \sqrt{\bar{\omega}_{n}}\right)
\end{aligned}
$$




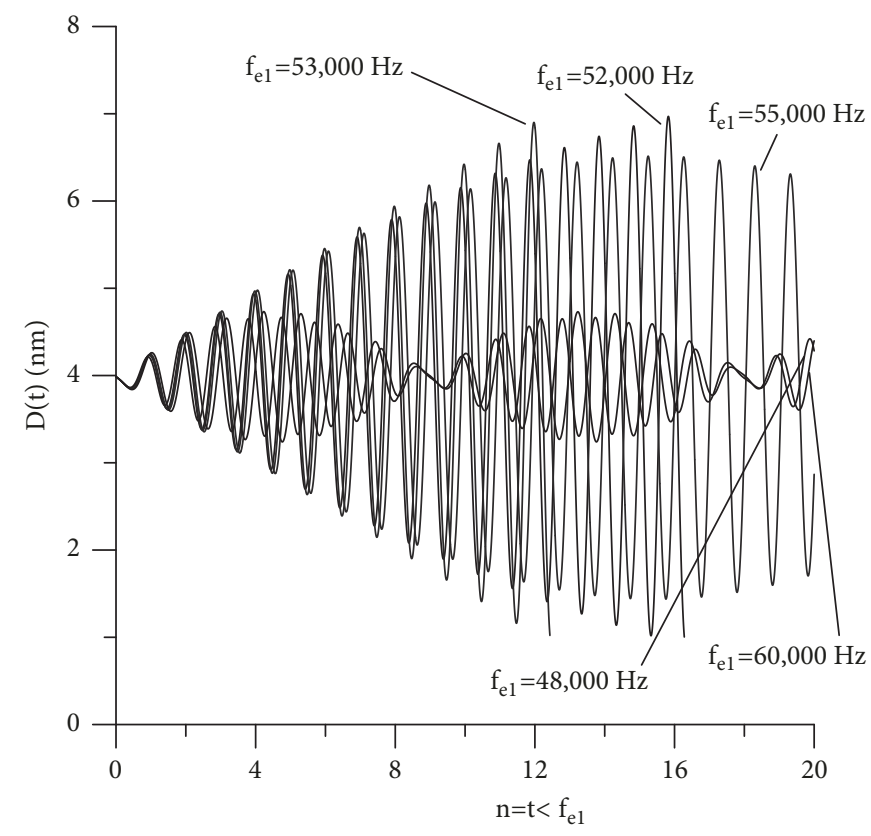

(a) $\left[W_{e 1}=0.05 \mathrm{~nm}, W_{e 2}=0, D_{0}=4 \mathrm{~nm}\right]$

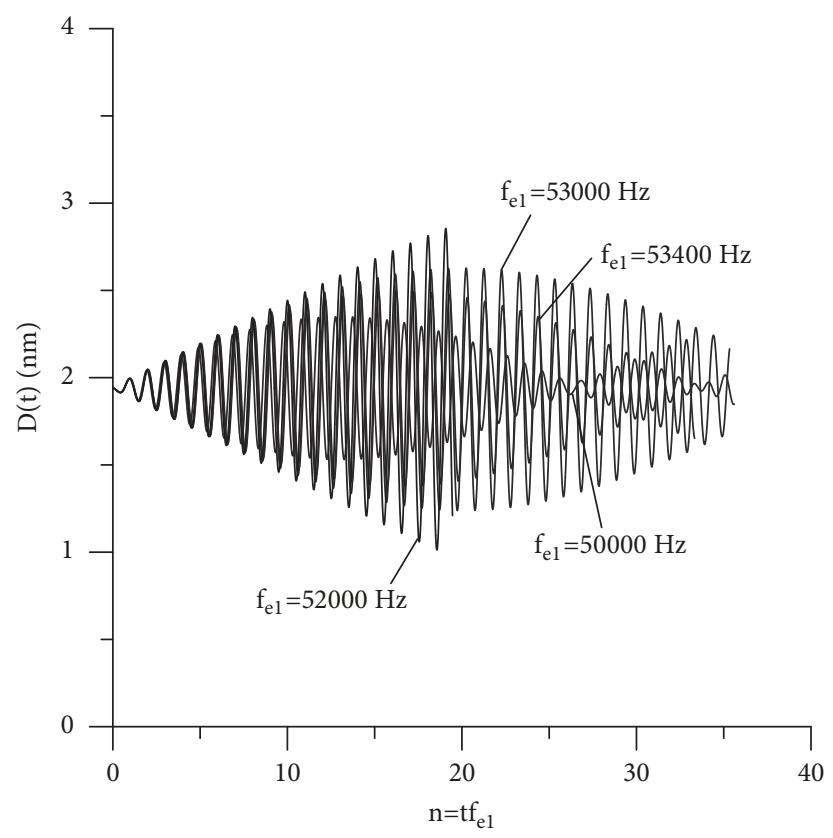

(b) $\left[W_{e 1}=0.01 \mathrm{~nm}, W_{e 2}=0, D_{0}=2 \mathrm{~nm}\right]$

FIGURE 2: The influences of the excitation frequency $f_{e 1}$, the excitation amplitude $W_{e 1}$, and tip-sample distance $D_{0}$ on the tip transient response $\left[A_{0}=10 \mathrm{~nm}, B=45 \mu \mathrm{m}, H=2.50 \mu \mathrm{m}, E=70.3 \times 10^{9} \mathrm{~Pa}, \rho=2.5 \times 10^{3} \mathrm{~kg} / \mathrm{m}^{3}, m_{t}=0.06723 \times 10^{-13} \mathrm{~kg}, L=200 \mu \mathrm{m}, k=1.5447 \mathrm{~N} / \mathrm{m}\right.$, and $f_{1}=$ $53525.9 \mathrm{~Hz}$,].

$$
\begin{aligned}
& \gamma_{n 1}=-\frac{1}{2 \bar{\omega}_{n}^{3 / 2}}\left[\left(\left(\bar{\omega}_{n}+2\right) \sinh \sqrt{\bar{\omega}_{n}}\right.\right. \\
& \left.-2 \sqrt{\omega_{n}} \cosh \sqrt{\bar{\omega}_{n}}\right)-\left(2 \sqrt{\bar{\omega}_{n}} \cos \sqrt{\bar{\omega}_{n}}\right. \\
& \left.\left.+\left(\bar{\omega}_{n}-2\right) \sin \sqrt{\bar{\omega}_{n}}\right)\right] \\
& \gamma_{n 2}=\frac{1}{6 \bar{\omega}_{n}^{2}}\left[\left(\left(\bar{\omega}_{n}^{3 / 2}+6 \sqrt{\bar{\omega}_{n}}\right) \sinh \sqrt{\bar{\omega}_{n}}\right.\right. \\
& \left.-\left(3 \bar{\omega}_{n}+6\right) \cosh \sqrt{\bar{\omega}_{n}}+6\right) \\
& -\left(\left(3 \bar{\omega}_{n}-6\right) \cos \sqrt{\bar{\omega}_{n}}+\left(\bar{\omega}_{n}^{3 / 2}-6 \sqrt{\bar{\omega}_{n}}\right) \sin \sqrt{\bar{\omega}_{n}}\right. \\
& +6)] \\
& \gamma_{n 3}=-\frac{1}{2 \bar{\omega}_{n}^{3 / 2}}\left[\left(\left(\bar{\omega}_{n}+2\right) \cosh \sqrt{\bar{\omega}_{n}}\right.\right. \\
& \left.-2 \sqrt{\bar{\omega}_{n}} \sinh \sqrt{\bar{\omega}_{n}}-2\right)-\left(2 \sqrt{\bar{\omega}_{n}} \sin \sqrt{\bar{\omega}_{n}}\right. \\
& \left.\left.-\left(\bar{\omega}_{n}-2\right) \cos \sqrt{\bar{\omega}_{n}}-2\right)\right] \\
& \gamma_{n 4}=\frac{1}{6 \bar{\omega}_{n}^{2}}\left[\left(\left(\bar{\omega}_{n}^{3 / 2}+6 \sqrt{\bar{\omega}_{n}}\right) \cosh \sqrt{\bar{\omega}_{n}}\right.\right. \\
& \left.-\left(3 \bar{\omega}_{n}+6\right) \sinh \sqrt{\bar{\omega}_{n}}\right)-\left(\left(3 \bar{\omega}_{n}-6\right) \sin \sqrt{\bar{\omega}_{n}}\right.
\end{aligned}
$$$$
\left.\left.-\left(\bar{\omega}_{n}^{3 / 2}-6 \sqrt{\bar{\omega}_{n}}\right) \cos \sqrt{\bar{\omega}_{n}}\right)\right] .
$$

The solution of (40) is

$$
\begin{aligned}
T_{n}(\tau)= & \cos \omega_{n}\left(\tau-\tau_{i}\right) T_{n}\left(\tau_{i}\right) \\
& +\frac{1}{\omega_{n}} \sin \omega_{n}\left(\tau-\tau_{i}\right) \frac{d T_{n}\left(\tau_{i}\right)}{d \tau} \\
& +\frac{1}{\omega_{n}} \int_{\tau_{i}}^{\tau} \sin \omega_{n}(\tau-\chi) \widetilde{F}_{n}(\chi) d \chi \\
\frac{d T_{m}(\tau)}{d \tau}= & -\omega_{m} \sin \omega_{m}\left(\tau-\tau_{i}\right) T_{m}\left(\tau_{i}\right) \\
& +\cos \omega_{m}\left(\tau-\tau_{i}\right) \frac{d T_{m}\left(\tau_{i}\right)}{d \tau} \\
& +\int_{\tau_{i}}^{\tau} \cos \omega_{m}(\tau-\chi) \widetilde{F}_{m}(\chi) d \chi
\end{aligned}
$$

Substituting (48a) and (48b) back into (22) results in the displacement of the cantilever. It should be noted that the tip displacement must satisfy condition (15).

2.3. Numerical Results of Transient Response and Discussion. The influences of the excitation frequency $f_{e 1}$, the excitation amplitude $W_{e 1}$, and tip-sample distance $D_{0}$ on the tip transient response are investigated and shown in Figures 2(a) and 2(b). Figure 2(a) demonstrates that the interacting distance is $\mathrm{D}_{0}=4 \mathrm{~nm}$. The initial displacement and velocity are 


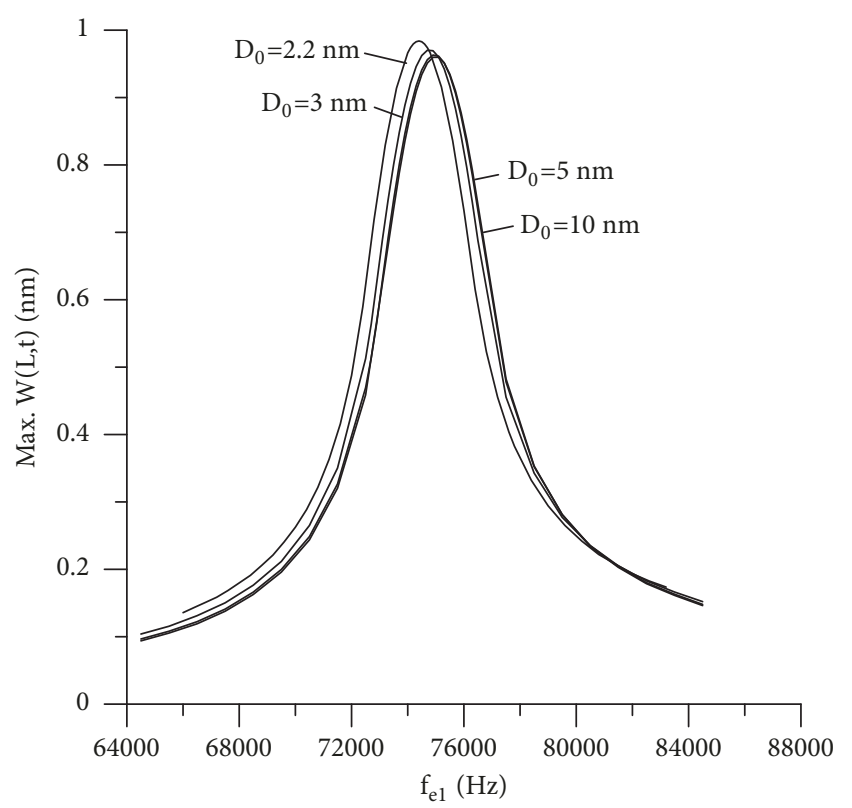

FIgURE 3: The influence of the distance between the tip and a sample surface on the tip response spectrum $\left[A_{0}=10 \mathrm{~nm}, B=45 \mu \mathrm{m}, \mathrm{H}=\right.$ $3.50 \mu \mathrm{m}, E=70.3 \times 10^{9} \mathrm{~Pa}, \rho=2.5 \times 10^{3} \mathrm{~kg} / \mathrm{m}^{3}, m_{t}=0.06723 \times 10^{-13} \mathrm{~kg}$, $L=200 \mu \mathrm{m}, k=4.2386 \mathrm{~N} / \mathrm{m}, f_{1}=74941.4 \mathrm{~Hz}, W_{e 1}=0.01 \mathrm{~nm}, W_{e 2}=0$, and $\mathrm{d} 0=3,5,10 \mathrm{~nm}]$.

zero and are listed in (13). One has the natural frequency of the cantilever without the interacting force, $f_{1}=53525.9 \mathrm{~Hz}$. When the frequency of root excitation is far from the natural frequency such as 48000 and $60000 \mathrm{~Hz}$ the small vibration of the cantilever is harmonically excited. When the frequency of excitation such as $53000 \mathrm{~Hz}$ approaches the natural frequency $f_{1}$, the vibration response is significantly increased. It is obvious that the frequency shift occurs due to the interacting force. Figure 2(b) demonstrates that the interacting distance is $\mathrm{D}_{0}=2 \mathrm{~nm}$. When the frequency of excitation is $52000 \mathrm{~Hz}$, the vibration response is significantly increased. It is obvious from Figures 2(a) and 2(b) that the smaller the interacting distance $\mathrm{D}_{0}$ is, the greater the frequency shift is.

Figure 3 demonstrates the influence of the distance between the tip and a sample surface on the tip response spectrum. It is shown that the smaller the interacting distance is, the larger the resonance frequency shift is.

Figures 4(a) and 4(b) demonstrate the influence of the size parameter $\delta$ on the tip response spectrum for $D_{0}=3$, $5 \mathrm{~nm}$. It is observed that the larger the size parameter $\delta$ is, the greater the frequency shift is. Moreover, the smaller the interacting distance $\mathrm{D}_{0}$ is, the greater the frequency shift is.

Further, the influence of two excitation modes on the tip response spectrum is investigated; Figure 5 demonstrates that if the second frequency of excitation $f_{e 2}=76500 \mathrm{~Hz}$ is far from the natural frequency $f_{1}=53525.9 \mathrm{~Hz}$, the response spectrum is as shown Figures 3 and 4 with one-mode excitation. Moreover, the influence of the interacting distance $D_{0}$ on the response spectrum is slight. However, if the second frequency of excitation $f_{e 2}=56500 \mathrm{~Hz}$ is close to the natural frequency $f_{1}=53525.9 \mathrm{~Hz}$, several resonant responses occur.
Moreover, the influence of the interacting distance $D_{0}$ on the response spectrum is significant. It is concluded that the response spectrum of two-mode excitations is significantly different to that of one-mode excitation.

\section{Prediction of Natural Frequencies}

3.1. The System with the Size Effect. The dimensionless governing equation and boundary conditions are

$$
(1+\delta) \frac{\partial^{4} w_{\delta}}{\partial \xi^{4}}+\frac{\partial^{2} w_{\delta}}{\partial \tau^{2}}=0
$$

The clamped boundary conditions are as follows.

At $\xi=0$ :

$$
\begin{aligned}
w_{\delta} & =0, \\
\text { and } \frac{\partial w_{\delta}}{\partial \xi} & =0
\end{aligned}
$$

At $\xi=1$ :

$$
\begin{gathered}
\frac{\partial^{2} w_{\delta}}{\partial \xi^{2}}=0 \\
(1+\delta) \frac{\partial^{3} w_{\delta}}{\partial \xi^{3}}-\mu_{t i p} \frac{\partial^{2} w_{\delta}}{\partial \tau^{2}}=f_{v \delta}
\end{gathered}
$$

where $f_{v \delta}=-c_{v \delta} / 6 d_{\delta}^{2}$ and $c_{v \delta}=A_{H} R_{\delta} L^{3} / E I L_{0}^{3}$, and the tipsurface distance is $d_{\delta}=1-w_{\delta}(1, \tau)$.

(A) Solution Method. Assume

$$
w_{\delta}(\xi, \tau)=\bar{w}_{\delta}(\xi) \cos \omega_{\delta} \tau,
$$

Substituting (54) into (49), one obtains

$$
\frac{d^{4} \bar{w}_{\delta}}{d \xi^{4}}-\bar{\omega}_{\delta}^{2} \bar{w}_{\delta}=0
$$

where $\bar{\omega}_{\delta}=\omega_{\delta} / \sqrt{1+\delta}$. Substituting (54) into the boundary conditions (50)-(52), one obtains the following.

At $\xi=0$ :

$$
\begin{aligned}
\bar{w}_{\delta} & =0, \\
\text { and } \frac{d \bar{w}_{\delta}}{d \xi} & =0
\end{aligned}
$$

At $\xi=1$ :

$$
\frac{d^{2} \bar{w}_{\delta}}{d \xi^{2}}=0
$$

Substituting (54) into the boundary conditions (53) and multiplying it by $\cos \omega \tau$ and integrating it from 0 to the period T, $2 \pi / \omega$, (53) becomes

$$
\frac{d^{3} \bar{w}_{\delta}}{d \xi^{3}}+\mu_{t i p} \bar{\omega}_{\delta}^{2} \bar{w}_{\delta}=\frac{-\bar{c}_{v \delta} \bar{w}_{\delta}(1)}{3\left[1-\bar{w}_{\delta}^{2}(1)\right]^{3 / 2}}
$$

where $\bar{c}_{v \delta}=c_{v \delta} / 1+\delta$. Further, the semianalytical solution can be determined by using the method by Lin [2] 


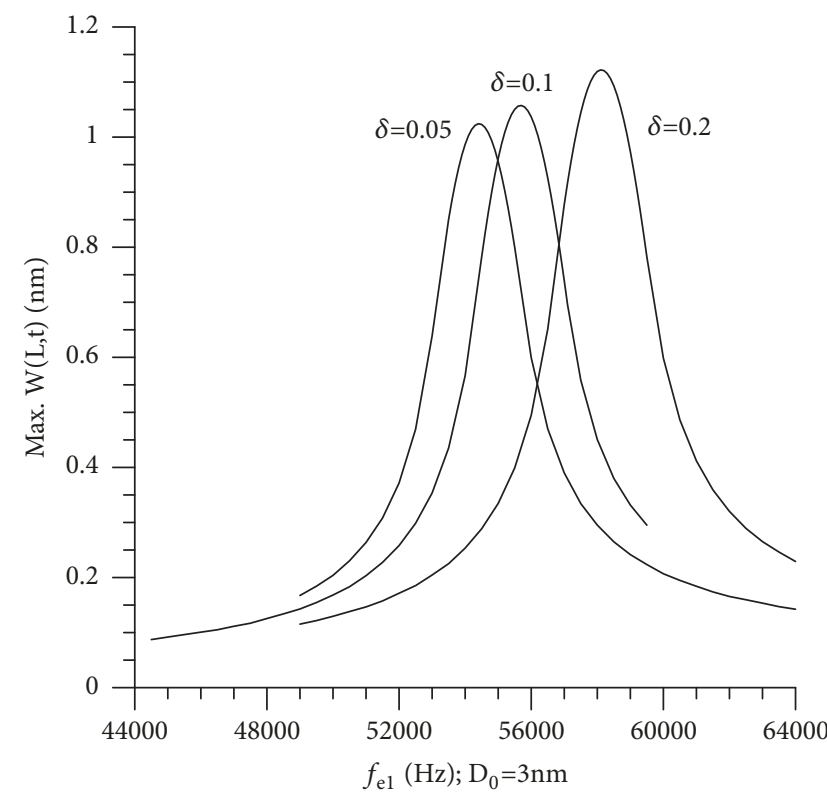

(a)

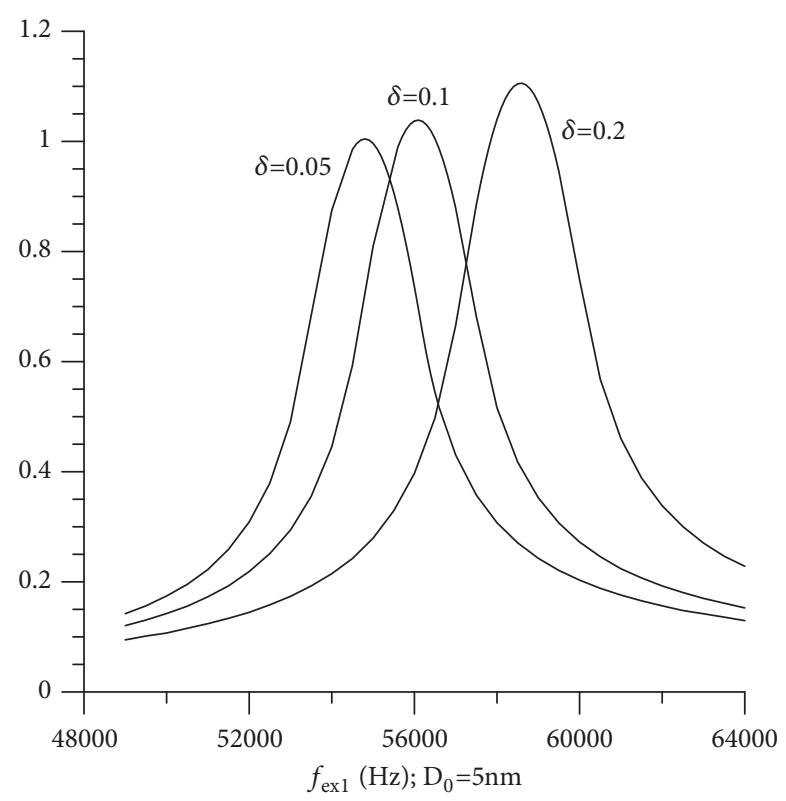

(b)

Figure 4: The influence of the size parameter $\delta$ on the tip response spectrum $\left[A_{0}=10 \mathrm{~nm}, B=45 \mu \mathrm{m}, H=2.50 \mu \mathrm{m}, E=70.3 \times 10^{9} \mathrm{~Pa}, \rho=\right.$ $2.5 \times 10^{3} \mathrm{~kg} / \mathrm{m}^{3}, m_{t}=0.06723 \times 10^{-13} \mathrm{~kg}, L=200 \mu \mathrm{m}, k=1.5447 \mathrm{~N} / \mathrm{m}, f_{1}=53525.9 \mathrm{~Hz}, \mathrm{~W}_{1 \mathrm{e}}=0.01 \mathrm{~nm}$, and $\left.\mathrm{W}_{2 \mathrm{~b}}=0.0 \mathrm{~nm}\right]$.

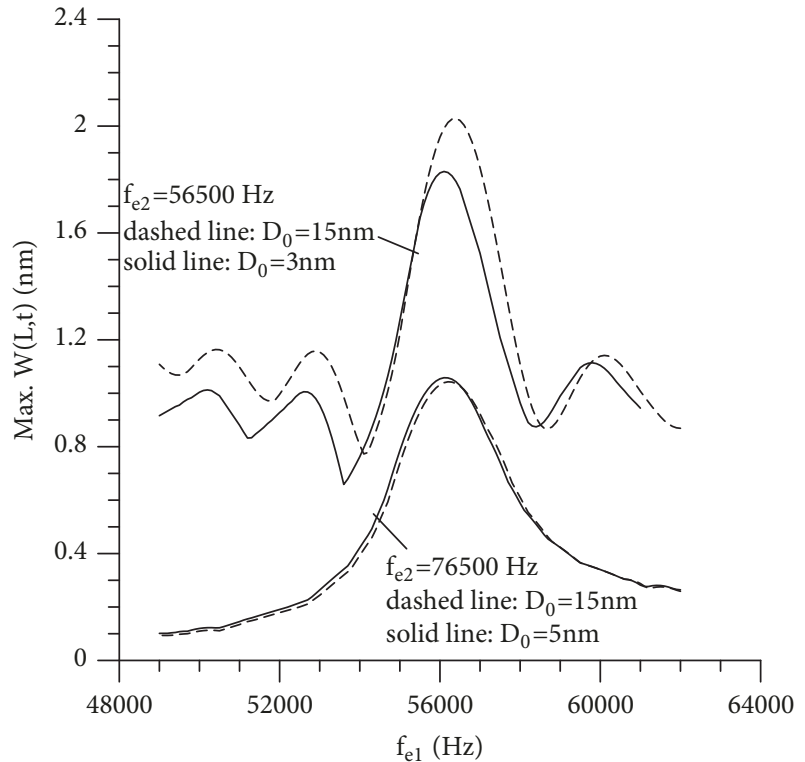

Figure 5: The influence of the two-mode excitation on the tip response $\left[\delta=0.1, A_{0}=10 \mathrm{~nm}, B=45 \mu \mathrm{m}, H=2.50 \mu \mathrm{m}, E=\right.$ $70.3 \times 10^{9} \mathrm{~Pa}, \rho=2.5 \times 10^{3} \mathrm{~kg} / \mathrm{m}^{3}, m_{t}=0.06723 \times 10^{-13} \mathrm{~kg}, L=200$ $\mu \mathrm{m}, k=1.5447 \mathrm{~N} / \mathrm{m}, f_{1}=53525.9 \mathrm{~Hz}$, and $\left.\mathrm{W}_{1 \mathrm{e}}=\mathrm{W}_{2 \mathrm{e}}=0.01 \mathrm{~nm}\right]$.

3.2. The Classical System without the Size Effect. The dimensionless governing equation and boundary conditions are

$$
\frac{\partial^{4} w_{0}}{\partial \xi^{4}}+\frac{\partial^{2} w_{0}}{\partial \tau^{2}}=0
$$

The clamped boundary conditions are as follows.
At $\xi=0$ :

$$
\begin{aligned}
w_{0} & =0, \\
\text { and } \frac{\partial w_{0}}{\partial \xi} & =0
\end{aligned}
$$

At $\xi=1$ :

$$
\begin{aligned}
\frac{\partial^{2} w_{0}}{\partial \xi^{2}} & =0 \\
\frac{\partial^{3} w_{0}}{\partial \xi^{3}}-\mu_{t i p} \frac{\partial^{2} w_{0}}{\partial \tau^{2}} & =f_{v 0}
\end{aligned}
$$

where $f_{v 0}=-c_{v 0} / 6 d^{2}$ and $c_{v 0}=A_{H} R_{0} L^{3} / E I L_{0}^{3}$, and the tipsurface distance is $d_{0}=1-w_{0}(1, \tau)$.

(A) Solution Method. Assume

$$
w_{0}(\xi, \tau)=\bar{w}_{0}(\xi) \cos \omega_{0} \tau,
$$

Substituting (65) into (60), one obtains

$$
\frac{d^{4} \bar{w}_{0}}{d \xi^{4}}-\omega_{0}^{2} \bar{w}_{0}=0
$$

Substituting (65) into the boundary conditions (61)-(63), one obtains the following.

$$
\text { At } \xi=0 \text { : }
$$$$
\bar{w}_{0}=0 \text {, }
$$

$$
\text { and } \frac{d \bar{w}_{0}}{d \xi}=0
$$


TABLE 1: Effects of the interacting distance $D_{0}$, the axial deformation, and the size dependency parameter $\delta$ on the first frequency [SiO2: $E$ $=70.3 \times 10^{9} \mathrm{~Pa}, \rho=2.5 \times 10^{3} \mathrm{~kg} / \mathrm{m}^{3}, B=45 \mu \mathrm{m}, H=3.5 \mu \mathrm{m}, L=200 \mu \mathrm{m}, R=1.5 \times 10^{-7} \mathrm{~m}, A_{H}=10^{-19}$ Joule, and $m_{\text {tip }}=3.18 \times 10^{-13} \mathrm{~kg}$; the first natural frequency of beam with $\delta=0, f_{1}=74355.803 \mathrm{~Hz}$, and $\left.\bar{W}(L)=3 \mathrm{~nm}\right]$.

\begin{tabular}{|c|c|c|c|c|}
\hline \multirow[t]{2}{*}{$\begin{array}{l}\mathrm{D}_{0} \\
(\mathrm{~nm})\end{array}$} & \multirow{2}{*}{$\delta=0.0$} & \multicolumn{2}{|l|}{$f_{1}(\mathrm{~Hz})$} & \multirow[t]{2}{*}{$\begin{array}{c}\text { Error } \\
(\%)\end{array}$} \\
\hline & & Semianalytical method & Based on (71) & \\
\hline 5 & 73686.61 & 77347.30 & 77283.17 & 0.0829 \\
\hline 6 & 74051.34 & 77694.80 & 77665.68 & 0.0375 \\
\hline 7 & 74187.16 & 77824.20 & 77808.15 & 0.0206 \\
\hline 8 & 74251.28 & 77885.32 & 77875.40 & 0.0127 \\
\hline 9 & 74286.01 & 77918.47 & 77911.82 & 0.0085 \\
\hline 10 & 74306.66 & 77938.20 & 77933.48 & 0.0061 \\
\hline 11 & 74319.82 & 77950.69 & 77947.28 & 0.0044 \\
\hline 12 & 74328.64 & 77959.10 & 77956.54 & 0.0033 \\
\hline 13 & 74334.81 & 77964.93 & 77963.01 & 0.0025 \\
\hline 14 & 74339.14 & 77969.18 & 77967.55 & 0.0021 \\
\hline 15 & 74342.38 & 77972.18 & 77970.95 & 0.0016 \\
\hline 20 & 74350.29 & 77979.84 & 77979.24 & 0.0008 \\
\hline 40 & 74354.29 & 77983.50 & 77983.43 & 0.0001 \\
\hline 80 & 74355.73 & 77984.95 & 77984.95 & 0.0000 \\
\hline
\end{tabular}

At $\xi=1$ :

$$
\frac{d^{2} \bar{w}_{0}}{d \xi^{2}}=0
$$

Substituting (65) into the boundary conditions (64) and multiplying it by $\cos \omega \tau$ and integrating it from 0 to the period $\mathrm{T}, 2 \pi / \omega,(64)$ becomes

$$
\frac{d^{3} \bar{w}_{0}}{d \xi^{3}}+\mu_{t i p} \omega_{0}^{2} \bar{w}_{0}=\frac{-c_{\nu 0} \bar{w}_{0}(1)}{3\left[1-\bar{w}_{0}^{2}(1)\right]^{3 / 2}}
$$

Further, the semianalytical solution can be determined by using the method by Lin [2].

3.3. Relation between Natural Frequencies and Size Parameter. It is discovered that if the following relations are satisfied

$$
\begin{aligned}
\bar{\omega}_{\delta} & =\omega_{0} \\
\text { or } \frac{\omega_{\delta}}{\sqrt{1+\delta}} & =\omega_{0} \\
c_{v 0} & =\bar{c}_{v \delta} \\
\text { or } \frac{A_{H} R_{0} L^{3}}{E I L_{0}^{3}} & =\frac{A_{H}\left(R_{\delta} /(1+\delta)\right) L^{3}}{E I L_{0}^{3}}
\end{aligned}
$$

the system composed of (55)-(59) is similar to that composed of (66)-(70) and $\bar{w}_{0}=\bar{w}_{\delta}$.

In other words, if the parameters $\left\{E, I, L, L_{0}\right\}$ of the two systems are the same, the tip radius relation $R_{0}=R_{\delta} /(1+\delta)$ and $\bar{w}_{0}=\bar{w}_{\delta}$, the relation of natural frequencies (71) must be satisfied.
Tables 1 and 2 demonstrate the comparison of the first two natural frequencies determined by using the analytical method $[2,4]$ and formula (71). It is found that these results are significantly consistent. When the interacting distance is large, the results are the same. When the interacting distance $\mathrm{D}_{0}=5 \mathrm{~nm}$, the errors of the first two frequencies are $0.08 \%$ and $0.002 \%$, respectively.

\section{Conclusion}

The transient response of AFM probe subjected to two-mode excitations based on the modified couple stress theory is investigated. The semianalytical solution is presented. The similarity of the classical Bernoulli-Euler beam system and the system based on the modified couple stress theory is discovered. The relation between the natural frequencies of the two systems is $\omega_{\delta}=\omega_{0} \sqrt{1+\delta}$. Several trends about the response spectrum due to two-mode excitations are obtained as follows.

(1) The response spectrum of two-mode excitations is significantly different to that of one-mode excitation. If the two frequencies of excitation are close to the natural frequency, several resonant responses occur.

(2) The larger the size parameter $\delta$ is, the greater the frequency shift is.

(3) The smaller the interacting distance $\mathrm{D}_{0}$ is, the greater the frequency shift is.

\section{Data Availability}

All the data used to support the findings of this article are included within the article. 
TABLE 2: Effects of the interacting distance $D_{0}$, the axial deformation, and the size dependency parameter $\delta$ on the second frequency $f_{2}$ [SiO2: $E=70.3 \times 10^{9} \mathrm{~Pa}, \rho=2.5 \times 10^{3} \mathrm{~kg} / \mathrm{m}^{3}, B=45 \mu \mathrm{m}, H=3.5 \mu \mathrm{m}, L=200 \mu \mathrm{m}, R=1.5 \times 10^{-7} \mathrm{~m}, A_{H}=10^{-19} \mathrm{Joule}$, and $m_{\text {tip }}=3.18 \times 10^{-13} \mathrm{~kg}$; the first natural frequency of beam with $\delta=0, f_{1}=74355.803 \mathrm{~Hz}$, and $\left.\bar{W}(L)=3 \mathrm{~nm}\right]$.

\begin{tabular}{|c|c|c|c|c|}
\hline \multirow[t]{2}{*}{$\begin{array}{l}\mathrm{D}_{0} \\
(\mathrm{~nm})\end{array}$} & \multirow{2}{*}{$\delta=0.0$} & \multicolumn{2}{|l|}{$f_{2}(\mathrm{~Hz})$} & \multirow[t]{2}{*}{$\begin{array}{c}\text { Error } \\
(\%)\end{array}$} \\
\hline & & Semianalytical method & Based on (71) & \\
\hline 5 & 465927.01 & 488678.25 & 488668.37 & 0.0020 \\
\hline 6 & 465983.22 & 488731.79 & 488727.32 & 0.0009 \\
\hline 7 & 466004.20 & 488751.78 & 488749.33 & 0.0005 \\
\hline 8 & 466014.11 & 488761.27 & 488759.72 & 0.0003 \\
\hline 9 & 466019.52 & 488766.43 & 488765.40 & 0.0002 \\
\hline 10 & 466022.69 & 488769.43 & 488768.72 & 0.0001 \\
\hline 11 & 466024.77 & 488771.43 & 488770.90 & 0.0001 \\
\hline 12 & 466026.19 & 488772.76 & 488772.39 & 0.0001 \\
\hline 13 & 466027.02 & 488773.59 & 488773.26 & 0.0001 \\
\hline 14 & 466027.77 & 488774.26 & 488774.05 & 0.0000 \\
\hline 15 & 466028.27 & 488774.76 & 488774.57 & 0.0000 \\
\hline 20 & 466029.52 & 488775.93 & 488775.88 & 0.0000 \\
\hline 40 & 466030.18 & 488776.44 & 488776.58 & 0.0000 \\
\hline 80 & 466030.34 & 488766.74 & 488766.74 & 0.0000 \\
\hline
\end{tabular}

\section{Conflicts of Interest}

The authors declare that they have no conflicts of interest.

\section{Acknowledgments}

The support of the Ministry of Science and Technology of Taiwan, R. O. C., is gratefully acknowledged (Grant number: MOST 106-2221-E-168-005).

\section{References}

[1] F. J. Giessibl, "Advances in atomic force microscopy," Reviews of Modern Physics, vol. 75, no. 3, pp. 949-983, 2003.

[2] S.-M. Lin, "Analytical solutions of the frequency shifts of several modes in dynamic force microscopy subjected to AC electrostatic force," IEEE Transactions on Nanotechnology, vol. 6, no. 4, pp. 404-412, 2007.

[3] S.-M. Lin and C.-C. Lin, "Phase shifts and energy dissipations of several modes of AFM: Minimizing topography and dissipation measurement errors," Precision Engineering, vol. 33, no. 4, pp. 371-377, 2009.

[4] S.-M. Lin, C.-T. Liauh, W.-R. Wang, and S.-H. Ho, "Analytical solutions of the first three frequency shifts of AFM non-uniform probe subjected to the Lennard-Jones force," Ultramicroscopy, vol. 106, no. 6, pp. 508-515, 2006.

[5] S. M. Lin, "Energy dissipation and frequency shift of a dynamic force microscopy with structural damping," Ultramicroscopy, vol. 106, no. 6, pp. 516-524, 2006.

[6] S. M. Lin, C. T. Liauh, W. R. Wang, and S. H. Ho, "Analytical solutions of the frequency shifts of several modes in AFM scanning an inclined surface, subjected to the Lennard-Jones force," International Journal of Solids and Structures, vol. 44, no. 3-4, pp. 799-810, 2007.
[7] D. Kiracofe and A. Raman, "On eigenmodes, stiffness, and sensitivity of atomic force microscope cantilevers in air versus liquids," Journal of Applied Physics, vol. 107, pp. 033506-033515, 2010.

[8] S. Santos, K. R. Gadelrab, V. Barcons et al., "The additive effect of harmonics on conservative and dissipative interactions," Journal of Applied Physics, vol. 112, no. 12, pp. 124901-124908, 2012.

[9] A. F. Payam, "Dynamic modeling and sensitivity analysis of dAFM in the transient and steady state motions," Ultramicroscopy, vol. 169, pp. 55-61, 2016.

[10] S. Santos, K. Gadelrab, J. Font, and M. Chiesa, "Single-cycle atomic force microscope force reconstruction: resolving timedependent interactions," New Journal of Physics, vol. 15, article 083034, 2013.

[11] D. R. Sahoo, A. Sebastian, and M. V. Salapaka, "Harnessing the transient signals in atomic force microscopy," International Journal of Robust and Nonlinear Control, vol. 15, no. 16, pp. 805820, 2005.

[12] P. I. Chang, P. Huang, J. Maeng, and S. B. Andersson, "Local raster scanning for high-speed imaging of biopolymers in atomic force microscopy," Review of Scientific Instruments, vol. 82, no. 6, article 063703, 2011.

[13] Á. S. Paulo and R. García, "Tip-surface forces, amplitude, and energy dissipation in amplitude-modulation (tapping mode) force microscopy," Physical Review B: Condensed Matter and Materials Physics, vol. 64, article 193411, 2001.

[14] B. Anczykowski, B. Gotsmann, H. Fuchs, J. P. Cleveland, and V. B. Elings, "How to measure energy dissipation in dynamic mode atomic force microscopy," Applied Surface Science, vol. 140, no. 3-4, pp. 376-382, 1999.

[15] R. Garcia, C. J. Gómez, N. F. Martinez et al., "Identification of nanoscale dissipation processes by dynamic atomic force microscopy," Physical Review Letters, vol. 97, article 016103, 2006. 
[16] S. M. Lin, C. L. Tsai, and H. C. Lee, "Effects of size dependency and axial deformation on the dynamic behavior of an AFM subjected to van der Waals force based on the modified couple stress theory," Precision Engineering, vol. 56, pp. 203-210, 2019.

[17] J. R. Lozano and R. Garcia, "Theory of multifrequency atomic force microscopy," Physical Review Letters, vol. 100, article 076102, 2008.

[18] N. F. Martinez, S. Patil, J. R. Lozano, and R. Garcia, "Enhanced compositional sensitivity in atomic force microscopy by the excitation of the first two flexural modes," Applied Physics Letters, vol. 89, article 153115, 2006.

[19] M. D. Aksoy and A. Atalar, "Force spectroscopy using bimodal frequency modulation atomic force microscopy," Physical Review B: Condensed Matter and Materials Physics, vol. 83, article 075416, 2011.

[20] D. C. C. Lam, F. Yang, A. C. M. Chong, J. Wang, and P. Tong, "Experiments and theory in strain gradient elasticity," Journal of the Mechanics and Physics of Solids, vol. 51, no. 8, pp. 14771508, 2003.

[21] A. W. McFarland and J. S. Colton, "Role of material microstructure in plate stiffness with relevance to microcantilever sensors," Journal of Micromechanics and Microengineering, vol. 15, no. 5, pp. 1060-1067, 2005.

[22] Q. Ma and D. R. Clarke, "Size dependent hardness of silver single crystals," Journal of Materials Research, vol. 10, no. 4, pp. 853-863, 1995.

[23] J. S. Stölken and A. G. Evans, "A microbend test method for measuring the plasticity length scale," Acta Materialia, vol. 46, no. 14 , pp. $5109-5115,1998$.

[24] R. D. Mindlin and H. F. Tiersten, "Effects of couple-stresses in linear elasticity," Archive for Rational Mechanics and Analysis, vol. 11, no. 1, pp. 415-448, 1962.

[25] R. A. Toupin, "Elastic materials with couple-stresses," Archive for Rational Mechanics and Analysis, vol. 11, pp. 385-414, 1962.

[26] Y. T. Beni and M. Abadyan, "Use of strain gradient theory for modeling the size-dependent pull-in of rotational nano-mirror in the presence of molecular force," International Journal of Modern Physics B, vol. 27, no. 18, Article ID 1350083, 2013.

[27] K. Kiani, "Vibration analysis of elastically restrained doublewalled carbon nanotubes on elastic foundation subjected to axial load using nonlocal shear deformable beam theories," International Journal of Mechanical Sciences, vol. 68, pp. 16-34, 2013.

[28] R. Ansari, K. Hosseini, A. Darvizeh, and B. Daneshian, "A sixthorder compact finite difference method for non-classical vibration analysis of nanobeams including surface stress effects," Applied Mathematics and Computation, vol. 219, no. 10, pp. 4977-4991, 2013.

[29] F. Yang, A. C. M. Chong, D. C. C. Lam, and P. Tong, "Couple stress based strain gradient theory for elasticity," International Journal of Solids and Structures, vol. 39, no. 10, pp. 2731-2743, 2002.

[30] S. K. Park and X.-L. Gao, "Bernoulli-Euler beam model based on a modified couple stress theory," Journal of Micromechanics and Microengineering, vol. 16, no. 11, pp. 2355-2359, 2006.

[31] S. Kong, S. Zhou, Z. Nie, and K. Wang, "The size-dependent natural frequency of Bernoulli-Euler micro-beams," International Journal of Engineering Science, vol. 46, no. 5, pp. 427-437, 2008.

[32] M. Asghari, M. T. Ahmadian, M. H. Kahrobaiyan, and M. Rahaeifard, "On the size-dependent behavior of functionally graded micro-beams," Materials and Corrosion, vol. 31, no. 5, pp. 2324-2329, 2010.
[33] N. Togun and S. M. Bağdatll, "Investigation of the size effect in euler-bernoulli nano-beam using the modified couple stress theory," Celal Bayar University Journal Of Science, vol. 13, no. 4, pp. 893-899, 2017.

[34] R. A. Alashti and A. H. Abolghasemi, "A size-dependent bernoulli-euler beam formulation based on a new model of couple stress theory," International Journal of Engineering, vol. 27, no. 6, pp. 951-960, 2014.

[35] S. T. Pourashraf, R. Gholami, and M. A. Sahman SIAshrafi, "Size-dependent resonant frequency and flexural sensitivity of atomic force microscope micro-cantilevers based on the modified strain gradient theory," International Journal of Optomechatronics, vol. 9, no. 2, pp. 111-130, 2015.

[36] J. S. Rao, Advanced Theory of Vibration, John Wiley \& Sons (Asia), 1994. 


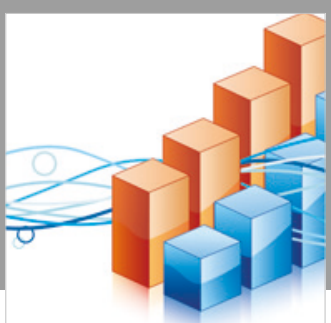

Advances in

Operations Research

\section{-n-m}
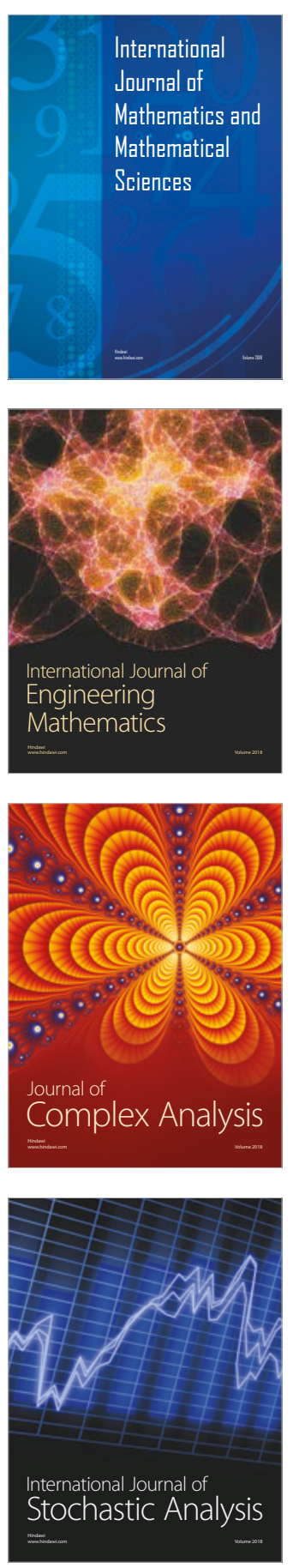
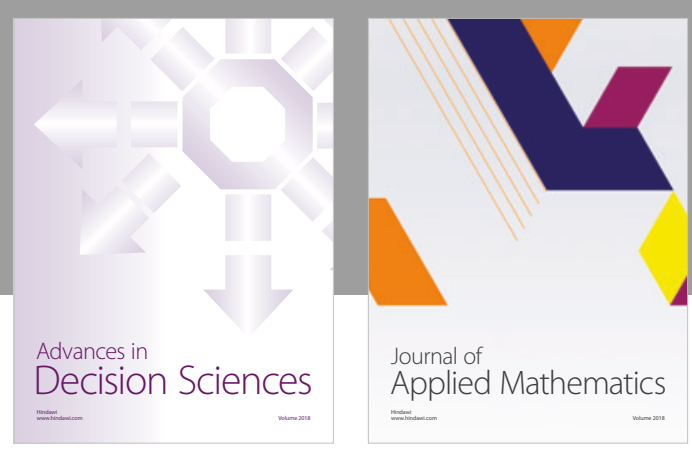

Journal of

Applied Mathematics
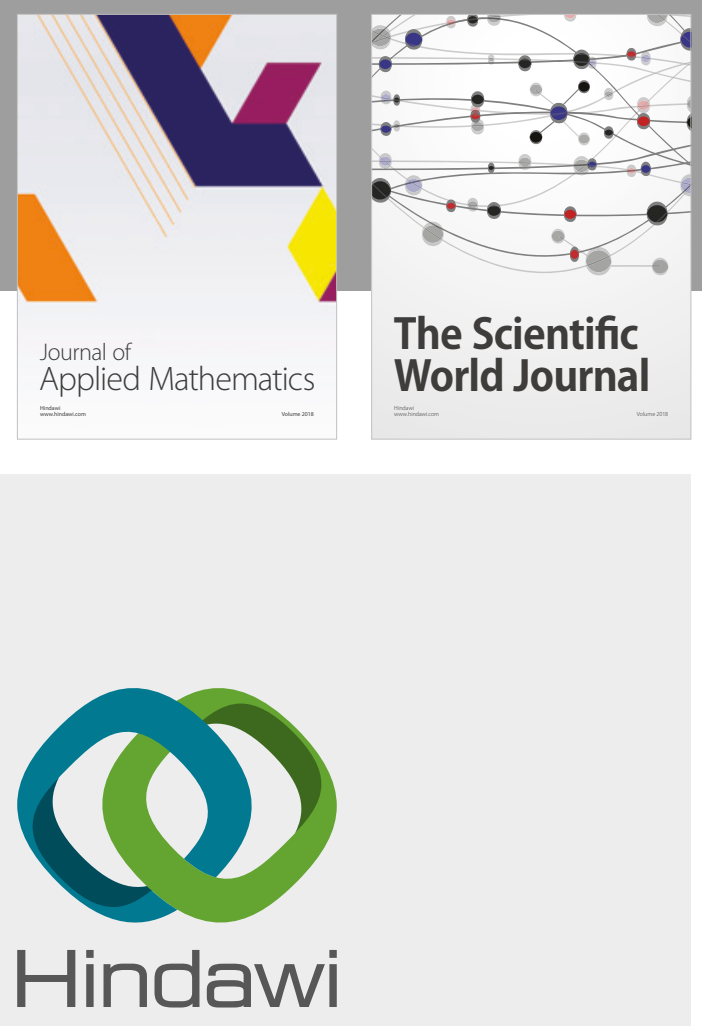

Submit your manuscripts at

www.hindawi.com

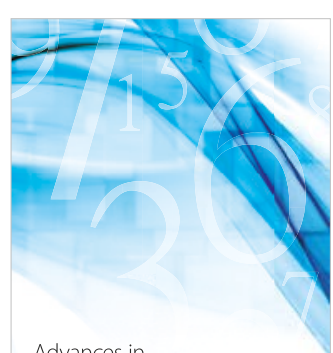

Advances in
Numerical Analysis
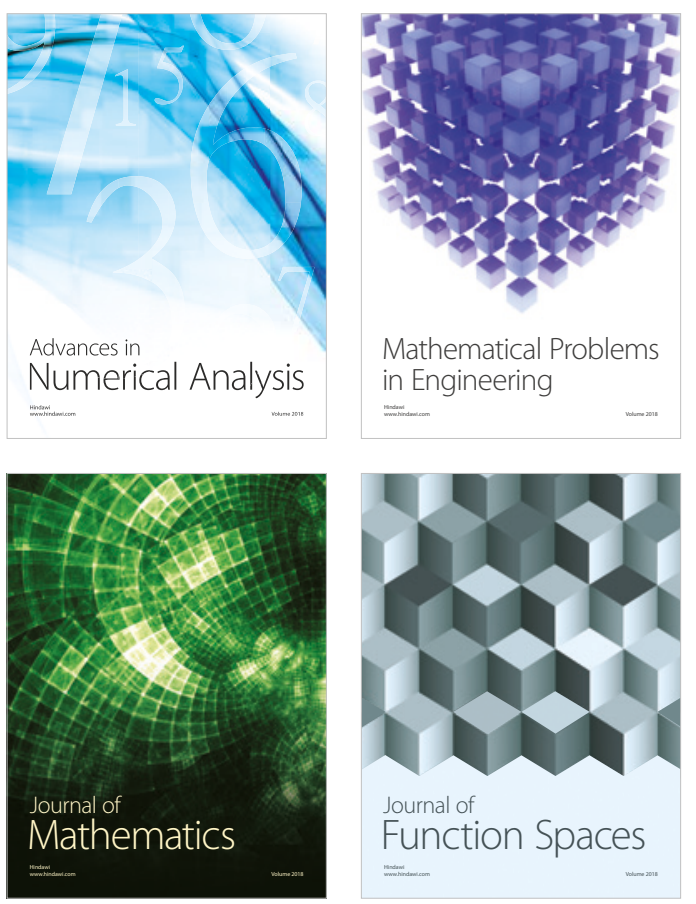

Mathematical Problems in Engineering

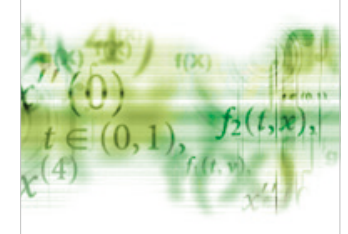

International Journal of

Differential Equations

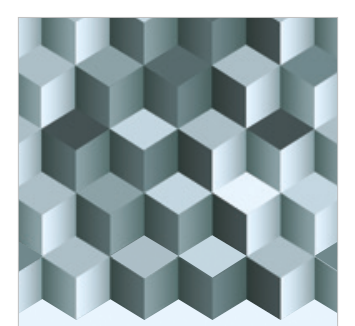

Journal of

Function Spaces

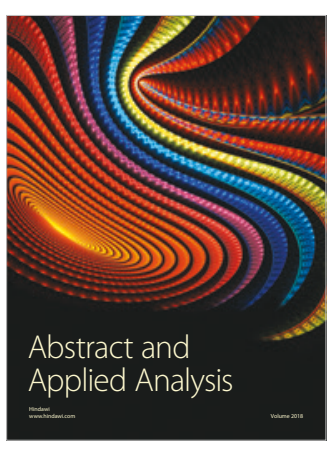

The Scientific

World Journal

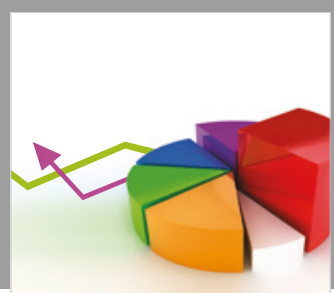

Journal of

Probability and Statistics
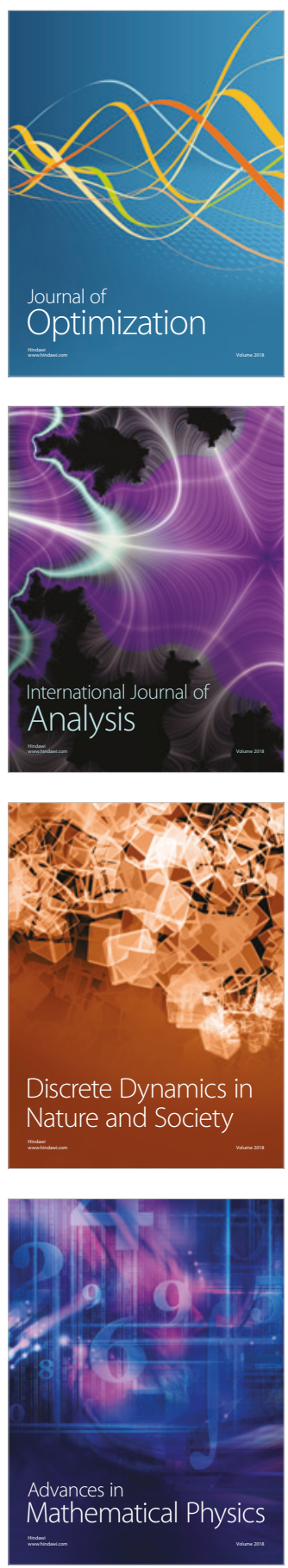